\title{
An ANN based Hyperspectral Waterway Control and Security System
}

\author{
Blanca Priego, Daniel Souto, Fernando Lopez Peña, Richard J. Duro \\ Integrated Group for Engineering Research \\ University of A Coruña \\ Ferrol, Spain \\ \{blanca.priego, dsouto, flop, richard\}@udc.es
}

\begin{abstract}
In this paper we report on some of the current advances in the development of Hywacoss (Hyperspectral waterway control and security system). The objective of the Hywacoss project is to produce a real time small, light and easy to transport visible and near infrared hyperspectral detection and recognition system that autonomously monitors waterways, especially port and bay areas, and detects and classifies all the traffic, producing alerts when previously unknown objects or behavior patterns arise. Obviously, Hywacoss involves dedicated hardware and software modules, some of them based on computational intelligence methods. Here we will provide a global description of the system and a detailed analysis of some of its modules, in particular those related to hyperspectral image segmentation and the Artificial Neural Network based spectralgeometrical identification and profiling subsystems.
\end{abstract}

Keywords- Hyperspectral image processing, Waterway control and security, Artificial Neural Networks.

\section{INTRODUCTION}

In recent years the security of ports, bays and waterways has become an issue of great concern [1][2]. Attacks on ships or installations (gas or fuel processing plants, storage depots, etc.) in these environments can result in very high economic and environmental costs. In fact, ships, especially container or passenger ships anchored near port facilities are sitting targets and very vulnerable. Additionally, disabling ships in waterways or ports may result in a complete stoppage of all the activity within their areas of influence for a long time. Thus, protecting these types of installations is of paramount importance.

In this line, we are currently working on a multisensory intelligent monitoring and protection system for ports and waterways called Watchman. The design of this system involves capturing real time information on what is going on in the area being monitored from multiple sensorial sources, both static and moving, and fusing this information to provide a view that is as complete as possible of all the activities that are taking place within the designated area where all the players are identified, tracked and profiled. Information may come from multiple buoys throughout the area that are sensing under water and on the surface through hydrophones, cameras, and other sensors, radar information obtained from shore or on moving boats, the location and identification sent in by ships, and information coming from remote sensing elements in the surroundings of the installation and from airborne platforms. It is one of these remote sensing systems, the Hywacoss hyperspectral imaging based system, that this paper will concentrate on.

A hyperspectral imaging system or hyperspectrometer typically obtains images in which the spectral information of every pixel is collected in hundreds of contiguous discrete spectral bands. As a result, each hyperspectral image contains a large amount of information that can be perceived as a cube with two spatial and one spectral dimensions. This detailed spectral information permits classifying different materials or targets with much higher accuracy and discriminative power than lower dimensional color representations.

In the original application of hyperspectrometers as a remote sensing instrument operated from high flying planes [3], hyperspectral images usually contained mixtures of spectra in every pixel as a consequence of the poor spatial resolution of the images. As these images were taken a large distance from the target, a single pixel would typically encompass over one hundred square meters of landcover. Given this problem, analysis methods were developed to provide the segmentation of the images in terms of the ratio of endmembers present in every pixel so as to improve the spatial discrimination of these systems when analyzing different types of covers. This is not the case of ground based applications [4][5]. In this type of applications, images are taken close enough to the subject to obtain a relatively detailed view; consequently, the problem is not always to extract subpixel information, but to classify as a function of multiple pixel combinations taking into account their geometric layout. In other words it is necessary to perform combined spatial-spectral classification processes.

This is the case of the hyperspectral data captured and processed by Hywacoss (HYperspectral WAterway COntrol and Security System). It is a ground based hyperspectral system that needs to perform three functions in an autonomous manner. On one hand, it must capture hyperspectral images, segment them and detect targets within them. After that, it selects the targets that were detected and identifies them using spatial-spectral information. Finally, it tracks these targets and obtains behavioral profiles that are used to detect suspicious or unexpected behaviors. In this paper we will concentrate on the first two functions as well as on the description of the instrument itself. 


\section{SENSOR SYSTEM}

Regarding the hardware part of the system, we have designed and constructed a small, light and easy to transport push-broom type spectrometer. It is shown in figure 1. It consists of a moving mirror that captures light that is focalized on a $10 \mathrm{~mm}$ long, $10 \mu \mathrm{m}$ wide slit that selects a single line from the image. The light corresponding to this pixel line is passed through a diffraction grid and its image is focalized on the sensing element of a front-illuminated interline CCD camera. This arrangement allows us to obtain 15.1 lines per second, each one of which contains 1392 pixels, where each pixel is represented using 1040 spectral bands in the 400-1000 nm wavelength interval (visible to near-infrared). This is fast enough for the application we are considering. The information obtained is directly sent to a processing computer for image processing and the other tasks required by the Hywacoss system.
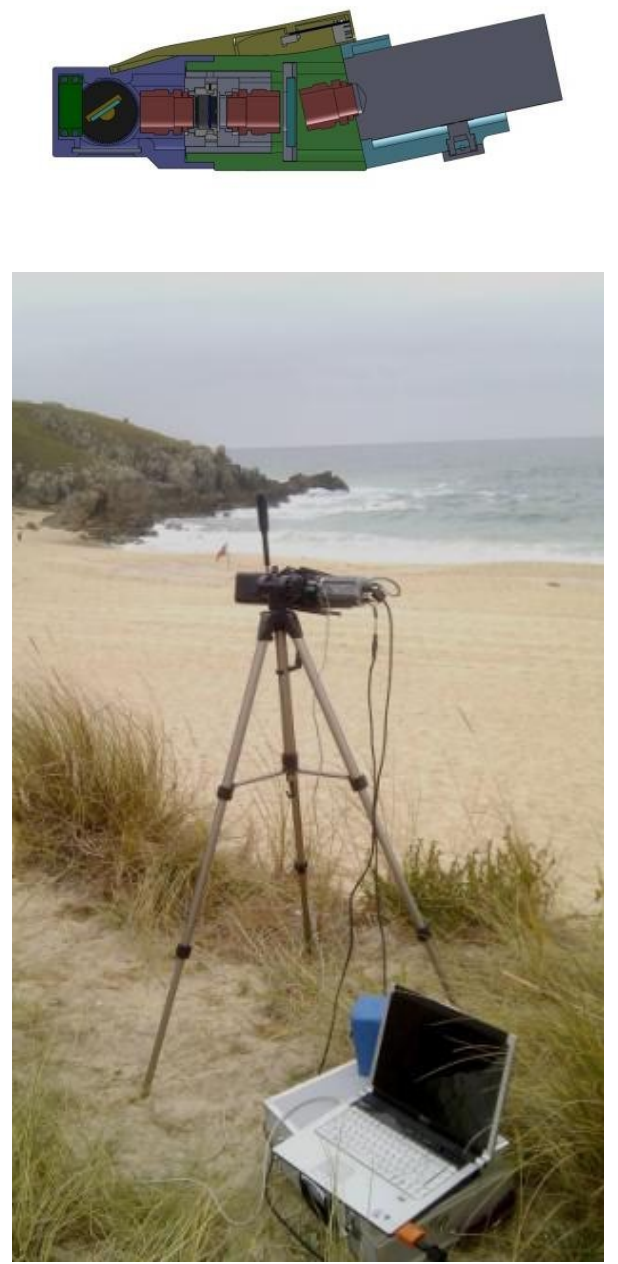

Figure 1: Schematic of the Hyperspectrometer designed and built for this project as well as view of the setup for its operation.
The hyperspectrometer is controlled by an Arduino processor that is in charge of controlling the mirror that sweeps the image and triggering the camera as well as communicating with the laptop that runs the image capture and storage software that was also developed within the project. The main design requirement when the system was created was for it to be easy to transport and operate while preserving the optimal optical characteristics. For this reason, all the elements were designed as small as possible and minimizing vigneting and other unwanted optical effects. Figure 1 displays a schematic of the hyperspectrometer as well as a picture of the arrangement used in the experiments presented in this paper where the small size and ease of deployment can be clearly appreciated.

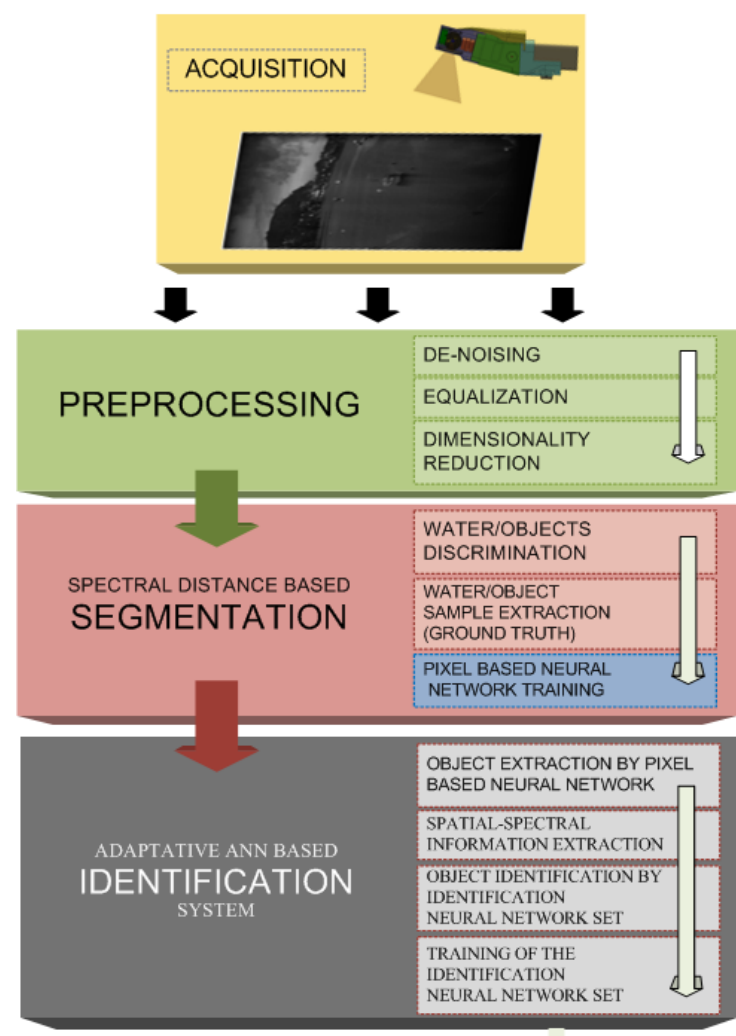

Figure 2: Diagram of the Hywacoss ship identification system

\section{HYWACOSS}

The part of the operation of Hywacoss that is described in this paper comprises three stages:

1- The spectrometer captures the images and an initial hyperspectral image preprocessing stage is carried out where the spectra of all the pixels are de-noised, equalized and normalized. A dimensionality reduction algorithm is also applied to remove unnecessary bands.

2- After this first stage, the image undergoes a spectral distance based segmentation process in which the areas corresponding to water and different objects in or around the water area are discriminated. This stage is used to train a pixel 
based classification process that permits improving the precision of the whole segmentation process.

3- A third processing stage takes the areas that are surrounded by water and not classified as water, extracts them from the image and identifies the object using an adaptive ANN based identification system.

Figure 2 displays a diagram of the whole process. In what follows we will provide a brief description of each stage.

\section{A.}

\section{Preprocessing}

This first stage comprises normalization and a dimensionality reduction processes. The normalization is carried out in order to compensate for intensity inhomogeneities due to the measurement system. On the other hand, dimensionality reduction permits reducing the number of bands that are necessary for subsequent processing. In fact, the objective is to use the minimum number of bands per pixel that allows the appropriate classification. In this case, the 1024 bands were binned to 64 which is the number of bands used to spectrally characterize each pixel in all subsequent stages.

\section{B. Segmentation and Classification}

Hyperspectral image classification and segmentation are similar and closely related problems. Following [6] when classifying $n$ pixels from a set $S$ of a hyperspectral image, each described by a d-dimensional feature vector, called spectral vector, $x \equiv\left(x_{1}, \ldots, x_{n}\right) \in R_{d} \times n$., we are assigning a label from a set $L \equiv\left\{L_{1}, \ldots, L_{K}\right\}$ of $K$ class labels that is inferred from the spectral vector. It is basically an injective function from $x$ to $L$. Segmentation, on the other hand, consists in the exhaustive partitioning of the input image into homogeneous regions with respect to some criterion, which, in this case, is the spectrum. In other words, to segment is to partition the set of image pixels $S$ into a collection of regions $R_{i} \subset S$, for $i=1$, $\ldots, K$, such that the image pixels in each set $R_{i}$ are spectrally close. Thus the main difference between classification and segmentation can be taken to be that the former does not use spatial information and the latter does. This use of spatial information tends to blur spectral differences between pixels and thus may sometimes hide information, especially when spectral changes occur spatially in a progressive manner. For this reason, here we have used a classification strategy in order to segment the hyperspectral image.

The main idea is to determine what parts of the images are water or other reasonably constant background and what parts are not and are thus possible targets. To this end we have trained an artificial neural network in order to classify each pixel as water or not water. The network chosen is a feedforward backpropagation trained network and its output can distinguish between pixels of water and pixels that correspond to other objects.
A distance based segmentation process was used to obtain the ground truth in order to train the pixel based classification network. To start with the distance based segmentation process, a pixel is randomly chosen and considered as the seed pixel of a new growing region. We select every neighboring pixel of the seed point for determining whether it belongs to the growing region by means of the application of a similarity rule. If a pixel satisfies the similarity rule, it is added to the growing region and later the similarity rule will be applied to all its neighboring pixels. The growing finishes when none of the neighboring pixels of the region satisfy the similarity rule.

Figure 3 displays the results of segmenting the previous image first considering a distance based segmentation algorithm and then using its results to train a segmentation ANN that is then used to segment the image pixel by pixel in order to obtain the final result.

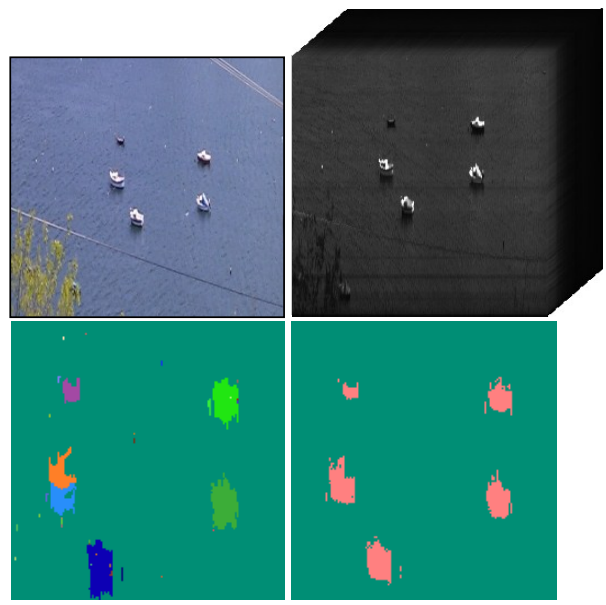

Figure 3: Top left: RGB image. Top Right: Hyperspectral cube. Bottom left: Example result of the distance based segmentation process. Bottom right: result of the pixel based classification process using a feed-forward backpropagation network with a hidden layer of 10 nodes

In order justify the choice of hyperspectral imaging and this segmentation procedure for its inclusion within the Security system as opposed to more traditional imaging techniques, we considered a scene that contained two sail ships as well as two other objects on the surface. This scene was captured as a hyperspectral cube and as a normal RGB image. Both images were processed in the same way and in Figure 4 we present the results of the segmentation process in both cases. It is clear to see that the segmentation resulting from the use of hyperspectral information is much cleaner than that of the RGB image. In fact, the RGB image segmentation presents so many artifacts that it is almost unusable. 

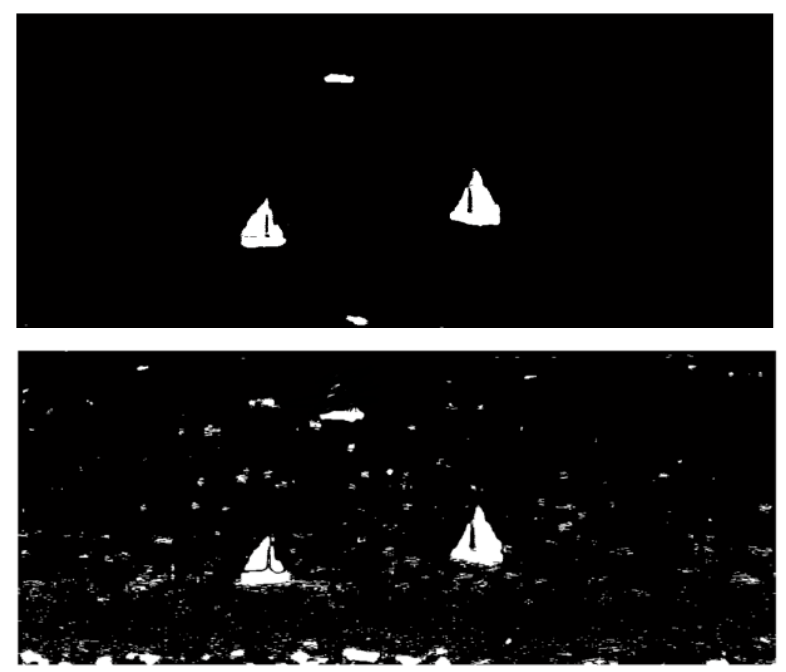

Figure 4: Pixel based segmentation of a hyperspectral image (top) and an RGB image (bottom) corresponding to the same scene.

\section{Artificial Neural Network based Identification}

Once the image has been segmented and all the non-water objects have been delimited, we start with the object identification stage. The main idea behind this stage is to identify and label ships or other moving objects that come into the port or bay area or move through the waterway. Some ships may come in regularly, others only once, but in principle they will all come in and at some point be identified either when they moor, or when asked by radio or through any other electronic or non-electronic means. However, a ship's hyperspectral image will not always be exactly the same even though it is still the same ship: the illumination of the area may be different (clouds, low sun, etc.), sails may be set, it may be loaded differently with containers, the orientation of the ship with respect to the hyperspectrometer may be different, etc. This implies that a system that can adapt and learn the main invariable traits of a given ship or object and their spectral/spatial variability in order to obtain ever more robust classifiers must be obtained.

To this end, we have created an identification system in two steps for the areas that are segmented out of the image (possible ships). There is a first step in which we take the segmented portion and group the spectra of the different pixels into ten clusters using a k-means clustering algorithm with a 1correlation measurement of the distance. The average spectrum of each cluster, the vertical location of their centers of mass and the percentage of pixels that belong to each one of them are taken as the spatial-spectral signature of the object.

The reason for choosing ten clusters is a compromise between the number of clusters and the variability of the spectra within them. If there are too few clusters, there is so much variability within them, especially as the ships must be identified no matter their angle with respect to the hyperspectrometer, that the clusters do not really reflect anything. On the other hand, as these cluster characteristics are the input data to the ANN based identification stage, the more clusters there are, the larger and harder to train the network will be. Ten clusters empirically turned out to be a good equilibrium.

The second step within the identification system is based on an ANN based identification stage. Within this step we find a pool of ANNs that act as ship detectors using as inputs the spatial-spectral signature extracted in the previous stage. As the spectra have been binned to 64 bands, the total number of inputs turns out to be 660 (ten spectra, ten centers and ten percentages). Each network has one hidden layer with 8 neurons and one output that indicates if the ship is the one corresponding to the network's label or not.

It is important to note here that in order to avoid problems when training the networks due to ambiguities in what is introduced through each input, as there is no way to establish a predefined order among the different clusters during the clustering process, we have decided to arrange the clusters in order of decreasing average value of their spectra. This implies that the first set of inputs are fed the spectrum, percentage and vertical position of the cluster represented by the spectrum with the largest average value, the second set of inputs that of the one with the second largest average value, and so on.
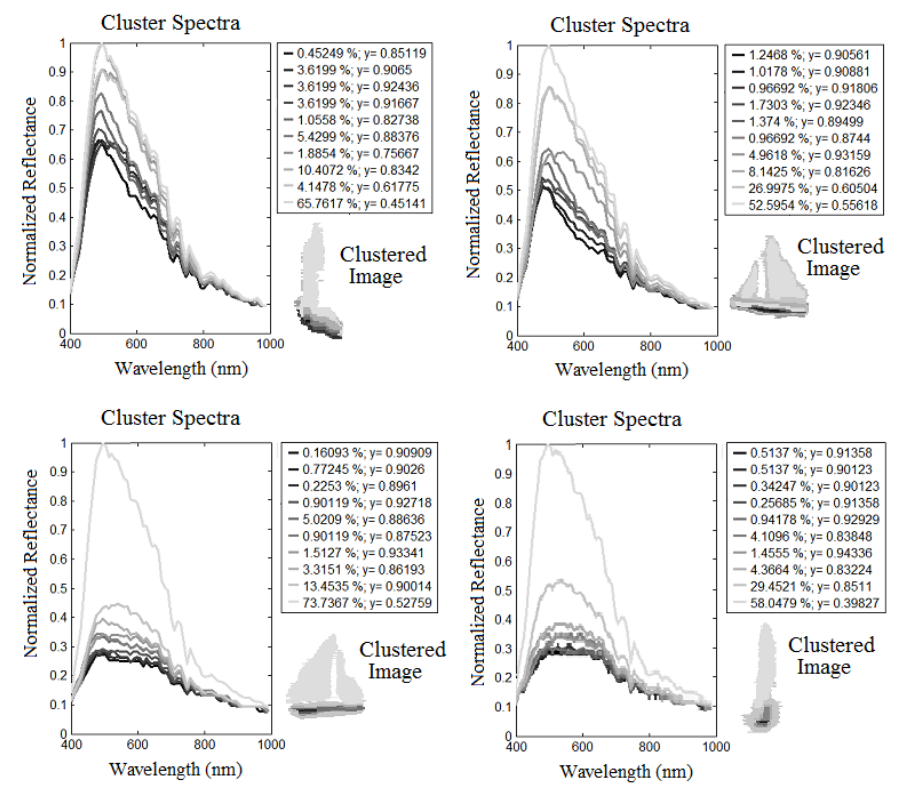

Figure 5: Results from clustering different ships in different orientations and spatial-spectral signature for each one of them including proportion of each spectral component.

Each ANN in the pool is specific for a particular ship and it is trained to detect this ship and ignore the rest. These networks continuously learn from the different spectral-spatial profiles that are being gathered from waterway activity. Thus the ANN pool is used to identify the object based on its spatial-spectral signature. If any of these ANN based detectors produces a positive result, the object is identified as the label of that ANN detector and its global spatial spectral signature is added to the positive training instances of the corresponding ANN. Otherwise the object is labeled as unknown, and an alarm message is produced for a human operator to follow it until it can be identified. Once the object has been identified, if it corresponds to a previously seen label, the global spectral- 
spatial signature is added to the spectral-spatial signature set of that object, if not, a new object is created and labeled. Finally, Whenever a new labeled spectral-spatial signature is acquired, a learning stage is run where all the networks in the pool (including the new randomly created ones corresponding to previously unknown objects that have now been labeled) are trained using the spectral-spatial signatures assigned to them as positive instances and those assigned to other objects as negative instances.

As more instances of objects are detected and identified, the system becomes better at identifying those it has already seen and differentiating them from those it has never seen before, thus improving its performance.

\section{EXPERIMENTAL RESULTS}

A series of experiments were run to validate the system and evaluate its performance. These experiments involved taking hyperspectral images of ships and boats in different situations, orientations and ambient circumstances, carrying out the procedure described in the previous sections in order to train the corresponding detectors and then testing them over different samples.

The training experiments can be divided into two classes. On one hand, we wanted to see how well the system was able to differentiate between a series of targets once enough information was available on all the targets. On the other, we deemed it important to evaluate the possible performance of the system in a more realistic setting where the samples of the ships or objects to be identified were not instantly available. That is, we tried to simulate the temporal aspects of traffic in a waterway, taking into account that the different samples of the targets would be progressively obtained and a large enough set of images of a given ship with different orientations and illuminations would only be compiled after weeks or months (depending on how often the ship went through the waterway).

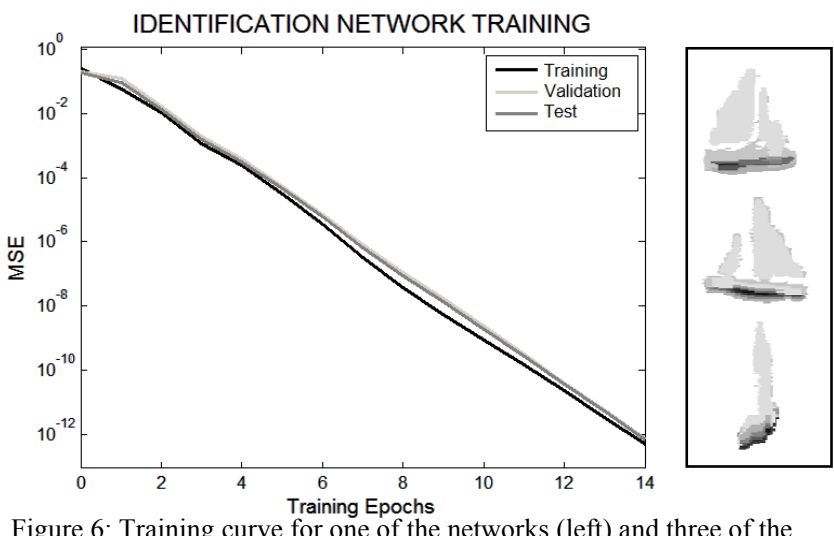

Figure 6: Training curve for one of the networks (left) and three of the orientations in the test set for that particular ship (right).

To perform an initial validation of the appropriateness of the spatial-spectral signature approach for the task of classifying and differentiating ships that can be seen at different angles and under different ambient circumstance we considered a set of hyperspectral images corresponding to two ships and some other elements. To make the problem a little more realistic, these images were segmented several times using different segmentation qualities. This was done basically to introduce noise that may occur during segmentation and thus evaluate the performance of the system under these conditions. A sample set was constructed for the networks containing 90 samples for each ship detector out of which 30 samples were positive and the rest negative. $80 \%$ of these samples were taken as training set and the remaining $20 \%$ as test set.

Figure 6 left displays the training curve for one of the networks and figure 6 right shows three of the orientations in the test set for that particular ship. It is clear that using the spatial-spectral signature that we constructed for each ship, the networks learn to identify the ship very fast with a very low

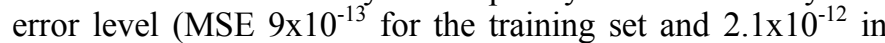
the case of the test set), which implies $100 \%$ identification of each ship, independently from its orientation.

The second experiment we present here involves the identification and differentiation of a set of ships in time as the waterway control system progressively acquires more information. In this case, we considered images of five boats in a waterway in the north of Spain. Images of these boats are presented in the top part of figure 7. Different images were acquired for these boats at different times and the Hywacoss system was executed as described in the previous section. That is, as new objects were identified they were included in the training set for the network pool (as positive training samples for the detector of the particular object and as negative training samples for the remaining networks in the pool). The results from this process can be seen in the confusion matrices above the top graph corresponding to three points in time of the process.

On the other hand, in the bottom part of Figure 7 the lower graph displays the evolution of the error of the identification networks for each one of the ships when applied to the identification of the ships detected in a given instant of time using the networks trained up to the previous instant of time. The $\mathrm{x}$-axis of the graph is discretized into instants of time in which images of ships are acquired. The labels indicate what ships went through the waterway in each particular instant. The other two graphs in the figure display the percentage of false positives and false negatives the system produces when a network trained up to a given instant of time is tested on the whole image set (including future images) in order to ascertain its generalization capabilities.

The results presented in the tables and in figure 7 are very promising. It is clear that Hywacoss learns to differentiate different ships or boats independently from angle or conditions. This classification improves significantly as new samples come in. In fact, after just a few views of the same ship (usually 2 or 3 ) the identification becomes quite reliable and generalizable to previously unseen views. This fact is confirmed if we take a look at the confusion matrices for three different instants of time. Initially, when the system has seen some ships only two times and some only once, there is quite a bit of confusion when testing over all of the rest of the images (remember that these images are images the system will see in the future). This confusion almost disappears after three different instances of some of the ships and the identification becomes perfect after four. 

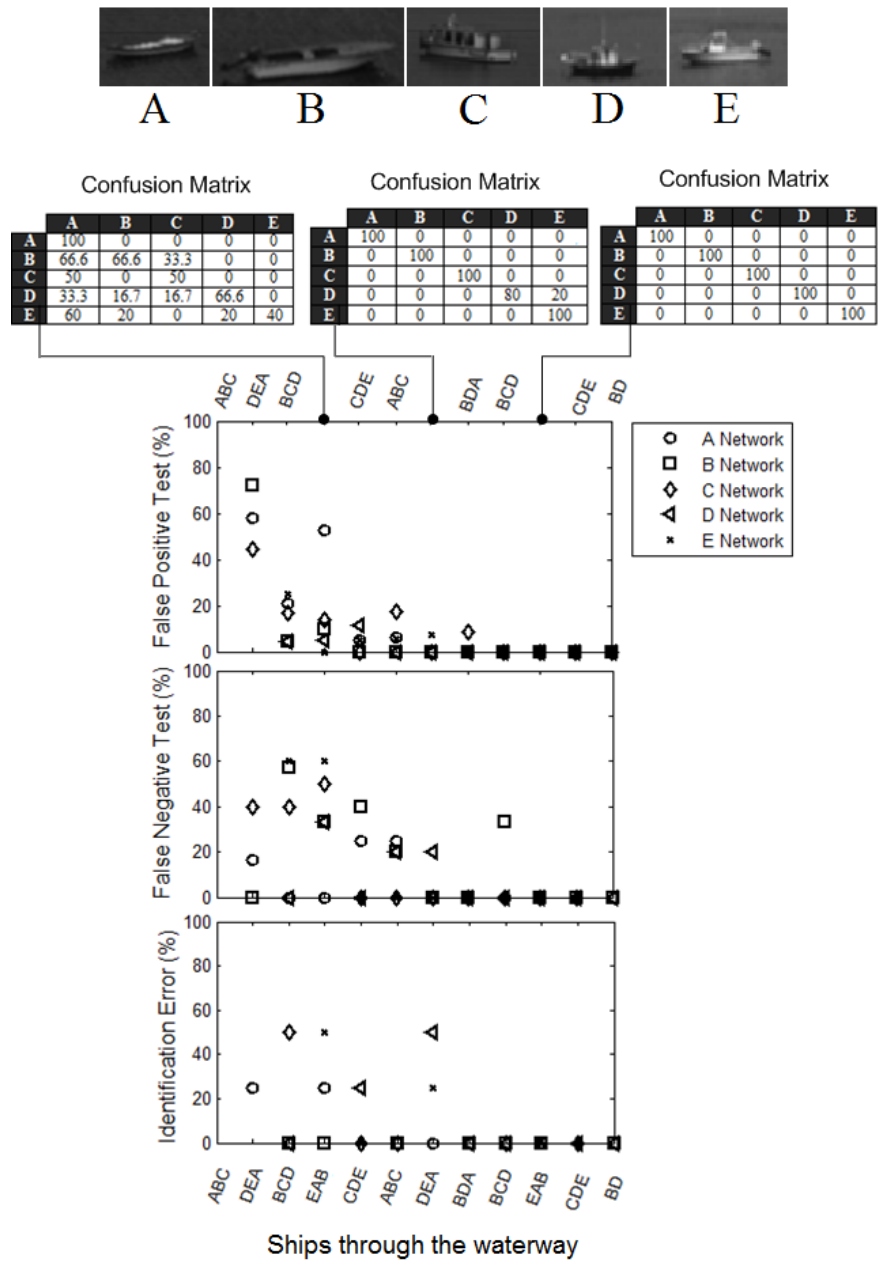

Figure 7: Images of some of the boats considered (top) and the evolution of the number of false positives and negatives produced by the identification networks when applied to the whole image set as time progresses and more training samples come in (top two graphs and confusion matrices) and identification error when identifying ships a given instant of time using the network trained up to the previous instant (bottom graph)

\section{CONCLUSIONS}

This paper has presented a hyperspectral based ship detection and identification system called Hywacoss to be deployed in ports and waterways that require this in order to establish control mechanisms or security measures. Hywacoss is based on a light and easy to transport and use hyperspectral system that was designed and constructed for this purpose. Its operation is based on a pool of ANNs that progressively learn the spatial-spectral signature of the ships they are presented with independently of variations in their angle with respect to the sensors and other changing circumstances. This is achieved thorough a three stage process that involves the hyperspectral segmentation of possible targets, the establishment of a profile for each target in terms of ten defining spectra and their position and proportion in the target and the creation of an ANN per identified target that associates the signature to a specific label.

The system has been tested on images of real ships and boats and the results have so far been very encouraging, confirming the descriptive capabilities of the spatial-spectral signature chosen for the problem and the appropriateness of the system structure. We are now in the process of compiling more extensive image libraries for particular port areas under different meteorological conditions in order to perform a better validation of the final systems.

\section{ACKNOWLEDGMENT}

This work was partially funded by the Xunta de Galicia and European Regional Development Funds through projects 09DPI012166PR and 10DPI005CT.

\section{REFERENCES}

[1] Frittelli, J. F., et al. (2003). Port and Maritime Security: Background and Issues. New York: Novinka Books.

[2] Helmick, J. S. (2007) "Port and Maritime Security: A Research Perspective". Journal of Transportation Security, V1, N1, pp. 15-28.

[3] Glackin D.L. and Peltzer G.R. (1999). "Civil, Commercial, and International Remote Sensing Systems and Geoprocessing”. Aerospace Press, American Institute of Aeronautics and Astronautics.

[4] Pan, Z.; Healey, G. E.; Prasad, M.; Tromberg, B. J. (2003) "Hyperspectral face recognition for homeland security". Infrared Technology and Applications XXIX. Edited by Andresen, B. F.; Fulop, G. F. Proceedings SPIE, V 5074, pp.767-776.

[5] de Juan, A.; Tauler, R.; Dyson, R.; Marcolli, C.; Rault, M.; Maeder, M (2004) "Spectroscopic imaging and chemometrics: a powerful combination for global and local sample analysis". Trends in Analytical chemistry, 23, pp. 70-79.

[6] Li, J., Bioucas-Dias, J. M., Plaza, A. (2010) "Semisupervised Hyperspectral Image Segmentation Using Multinomial Logistic Regression with Active Learning”. IEEE Transactions on Geoscience and Remote Sensing, V. 48, N.11, pp. 4085-4097.

[7] Robila S.A. (2004), "Using spectral distances for speedup in hyperspectral image processing", International Journal of Remote Sensing, V26, 24, pp. $5629-5650$. 